\title{
The Analysis of Fluid Pressure Impact on String Force and Deformation in Oil and Gas Wells
}

\author{
Gao Baokui, Ren Jingwei ${ }^{\text {a }}$ \\ Department of Petroleum Engineering, China University of Petroleum, Beijing \\ Beijing, China
}

\begin{abstract}
Fluid pressure is a crucial factor to tubular string strength and deformation in oil and gas wells, and it is the most difficult factor to deal with. When the string constrained by downhole tools, such as packers, action pattern of fluid on string is changed. Calculation methods of string stress and deformation given by engineering handbooks doesn't distinguish these issues in detail. So mistakes are often made when these methods are used. Tangled concepts lead to large calculation error. In this paper, the influence of fluid pressure on string axial force and deformation, buoyancy treatment in packed condition, are discussed roundly both in vertical wells and directional wells. Practical calculating method of string axial force through the hook load is presented, and element buoyancy in different borehole trajectory is given. It is found that the traditional simplified buoyancy coefficient method, which is used to calculate string axial force and axial extension, can only be used in vertical wells with tubular string suspended freely, because in this condition buoyancy acts on the bottom of string. If the string is constrained by downhole tools, such as packer or anchor, buoyancy could not be treated as usual. In directional well the buoyancy not only changes string axial force but induces shear stress in string cross section. When calculating the influence of fluid on string, operation sequence and constraints from borehole and downhole tools should be considered comprehensively.
\end{abstract}

\section{Background}

In complex structural wells, such as high-pressure-hightemperature (HPHT) wells, deep wells and directional wells, the prediction and control of tubular string axial force and axial deformation are very important to ensure working safety[1]. Mistakes made in design stage can lead to a lot of troubles in the following operations, even accidents. There are many factors that affect string deformation, including temperature, fluid pressure, tool weight, friction, well trajectory, constraints, string deformation, and so on[2,3]. Among them, the influence of fluid pressure on the string deformation is the most familiar one and, in the mean time, it is prone to being misunderstood.

The influence of fluid pressure on string acts in different aspects. First of all, the fluid pressure makes string axial force leap sharply at string bottom and tapered string steps due to the so-called piston effect. Secondly, fluid pressure leads to the axial strain by swelling effect. Thirdly, in the directional wells, fluid pressure causes shear stress in string cross section to increase the bending deformation. And finally, fluid pressure arouses string virtual axial force to cause string buckling, and buckled string leads to string shortening, contact force and friction with borehole.

\footnotetext{
a Ren Jingwei: rjw318318@126.com
}

Simple and practical calculation methods about liquid pressure effect can be found in various kinds of engineering handbooks. But the applicable conditions of the methods are not confined clearly, so mistakes are often made when they are used[4,5].

Downhole string axial force calculating method from hook load to bottom is presented. Fluid pressure change and its influence on string deformation in operation process is illuminated clearly, and the results will have great engineering value during complex string operation.

\section{String Axial Forces in Vertical Wells}

\subsection{Freely suspended string}

For a freely hung tubular string in a vertical borehole full filled with liquid, let $a, b$ and $H$ for inside radius, outside radius and length of string respectively, and $\gamma_{o}$ for the fluid density.

\subsubsection{Fluid inside and outside of string being connected}

In any well depth, the internal and external fluid pressure on string surface is equal, buoyancy acting on string is 


$$
F_{f}=\gamma_{o} H \pi\left(b^{2}-a^{2}\right)=\gamma_{o} H A_{s}
$$

Where $A_{s}=\pi\left(b^{2}-a^{2}\right)$ is cross sectional area of pipe wall.

Equation (1) shows that the buoyancy acting on string equals to fluid weight it displaced, the direction is upward. Despite fluid pressure exerts on any point of string surface, buoyancy actual acts on string bottom according to the principle of force composition.

Let $\gamma_{s}$ for the density of steel, so the suspending weight (hook load) of string is

$$
W=\left(\gamma_{s}-\gamma_{o}\right) H A_{s}
$$

Taking wellhead as origin, set coordinates system along well depth, and then axial force (tension is positive, similarly here in after) of string at any well depth is

$$
F_{x}=W-\gamma_{s} x A_{s}=\left[\gamma_{s}(H-X)-\gamma_{o} H\right] A_{S}
$$

When $x=\mathrm{H},\left.F_{x}\right|_{x=H}=-F_{f}=-\gamma_{o} H A_{s}$. Namely, buoyancy acts on string bottom end in vertical wells.

\subsubsection{The string being sealed at lower end}

Let $\gamma_{i}$ for the density of fluid in string, so the total buoyancy of string is

$$
F_{f}=\gamma_{o} H \pi b^{2}-\gamma_{i} H \pi a^{2}=\gamma_{o} H A_{o}-\gamma_{i} H A_{i}
$$

Where $A_{i}=\pi a^{2}$ and $A_{o}=\pi b^{2}$ is inner and outer area of string cross section respectively.

The hook load (suspending weight) of string is

$$
W=\gamma_{s} H A_{s}-\left(\gamma_{o} H A_{o}-\gamma_{i} H A_{i}\right)
$$

The axial force of string at any well depth is

$$
F_{x}=\gamma_{s} H A_{s}-\left(\gamma_{o} H A_{o}-\gamma_{i} H A_{i}\right)-\gamma_{s} x A_{s}
$$

At $x=\mathrm{H}$, the buoyancy is axial force.

\subsection{Bottom end packed string}

There are packers at the bottom of string in many circumstances[6,7,8], and sometimes the packer permits string bottom end axial movement, or slip joint is used to implement the movement. Hereinafter, only the case is discussed in which string bottom end axial movement is impossible.

From the above analysis it can be known that buoyancy actually acts on the bottom of string. But from the moment the packer is set, buoyancy effect is obstructed by packer, and string axial force has no direct relationship with fluid pressure below the packer. If temperature and pressure do not change before and after packer setting, Equation(3) and Equation (6) can still be used. But this is not the normal case. In fact, both temperature and pressure can change frequently, so new calculation method must be established which is more complicated than that above.

Assume hook load $F_{0}$ is known, string axial force distribution along the well depth is

$$
F_{x}=F_{0}-x \gamma_{s} A_{s}
$$

Attention must be paid that there is inevitable connection between the hook load and buoyant weight.

If the hook load cannot be obtained directly, then string axial force can only be speculated according to the initial length of string, the change in temperature and pressure before and after the packer setting. And the socalled floating method couldn't be used in the process.

\subsection{Piston force}

When tapered strings are used, steps appear inside and out-side string from the change in cross sections. A resultant axial force on each step, which is called piston force, leads the string axial force abrupt change. In fact, the piston force is part of buoyancy.

With tapered strings, let $A_{i u}$ and $A_{o u}$ for the inside and outside cross sectional area of upper string respectively, and $A_{i d}$ and $A_{o d}$ for that of lower string, let $P_{i}$ and $P_{o}$ for internal and external fluid pressure at junction point respectively. Therefore, the piston force at this point (upwards is positive, similarly here in after) is

$$
F_{p}=\left(A_{o u}-A_{o d}\right) P_{o}-\left(A_{i u}-A_{i d}\right) P_{i}
$$

If a control valve is employed in the string, then when the valve is closed, fluid pressure above and below it will be different, and so a piston force comes into being as

$$
F_{p}=A_{i}\left(P_{i d}-P_{i u}\right)
$$

Where $P_{i u}$ and $P_{i d}$ is fluid pressure above and below the valve respectively.

\subsection{Fluid pressure and buoyancy}

From the preceding analysis, only when a simple string under simple constraint, the buoyancy equals to the weight of fluid displaced by string. In fact, the socalled buoyancy is a resultant force of fluid pressure, and should not be simply described as the displaced fluid weight.

In the oil and gas wells, almost all strings can experience changes in fluid pressure. These changes may come from surface operation, formation pressure, thermal effect, diverse fluid density, and so on. So when calculating the influence of fluid pressure on string stress and deformation, all the factors must be taken in consideration and, in the mean time, constraints must be conformed to.

For example, if there exists wellhead pressure or annular pressure, the force the string obtained from fluid 
pressure should not be simply calculated from buoyant concept.

\section{String Deformation in Vertical Well}

\subsection{Deformation by axial force}

According to the relationship of axial stress and strain, string axial deformation can be calculated.

Generally string axial force is not a constant, so integral method is needed to obtain axial deformation.

In any well depth $\mathrm{x}$, intercept a infinitesimal section $d x$, its axial elongation is

$$
d(\Delta l)=\varepsilon_{x} d x=\frac{F_{x}}{A_{s} E} d x
$$

Where $\varepsilon_{x}$ is axial strain; $E$ is the Young's elastic modulus of steel.

This is the basic formula for calculating axial elongation of string. Total axial deformation could be obtained by integration along the string.

The calculation can be simplified by using the axial force at midpoint of string. But this is not a practical method because many mistakes can be made in the simplification process.

\subsection{Swelling effect}

In addition to the string axial force, fluid pressure can also induce axial deformation, this is socalled swelling effect. According to the basic theory of elasticity, swelling effect can be calculated from the following formula

$$
\varepsilon_{x}=\frac{2 \mu}{E} \cdot \frac{P_{o} R^{2}-P_{i}}{R^{2}-1}
$$

Where $\mu$ is the poisson's ratio of steel, $R$ is the ratio of OD/ID of the pipe.

This formula is obtained under the hypothesis of plane stress (string could free expansion at axial direction). Axial deformation caused by swelling effect can be calculated by integrating formula (11).

\subsection{Buckling effect}

Buckling is a common deformation phenomenon of oil and gas well string. The influence of fluid pressure on the string buckling lies in two aspects: one is the string axial force, the other is the so-called virtual axial force. Lubinski demonstrate through theoretical analysis and experiment that the effective axial force is the key factor that controlling string buckling. The string effective axial force $F_{e}$ (compression is positive) at any well depth $x$ is

$$
F_{e}=-F_{x}+P_{i} A_{i}-P_{o} A_{o}
$$

If the string is long enough, axial buckling condition

$$
F_{e} \geq F_{c r}
$$

Where $F_{c r}$ is the critical buckling force.

Because borehole size and string constraint are different, $F_{c r}$ have different values. On the other hand, post buckling shape is different. String buckling induces two aftermaths: string shortening and friction.

\section{String Axial Forces and Deformation in Deviated Wells}

In the deviated wells, impact of buoyancy on string is more difficult to investigate than in vertical wells. In most cases, precise calculation isn't practical, but sometimes theoretical analysis is necessary. In order to demonstrate this problem, take a curve string section to discuss, see Fig. 1a), where internal fluid pressure is ignored.

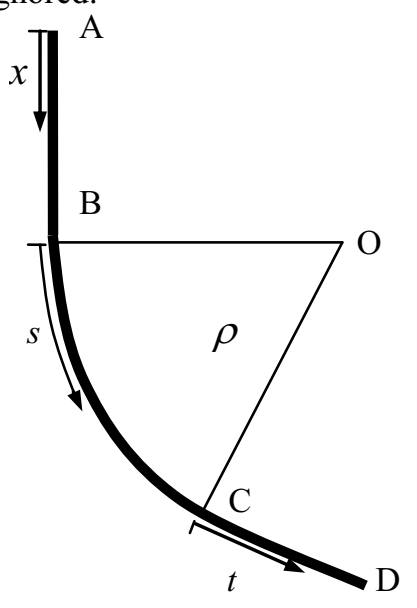

a) String Diagram

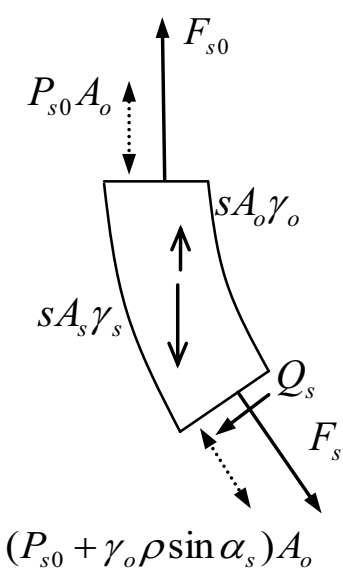

b) Bending Section

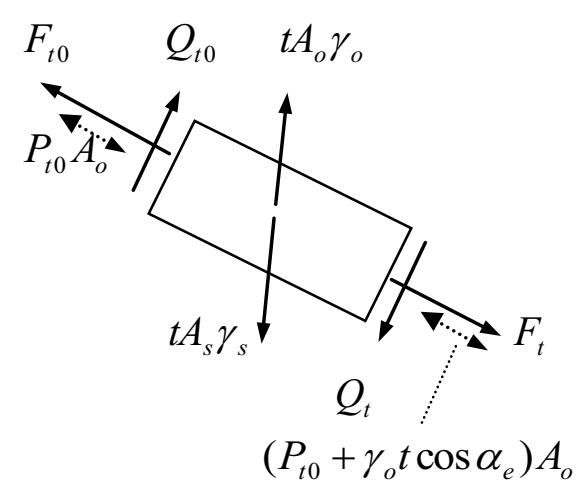

c) Deviated Section

Figure 1. String load analysis in directional well

For such a string section, it is troublesome to precisely calculate the fluid influence on axial force and deformation, and it is not necessary from engineering angle. So a simplified calculation is practical. In the 
process of simplification, attention should be paid to the concepts of fluid distribution and resultant force.

Assume that the hook load $W$ is known. Then string axial force distribution can be calculated in this way: Divide the string into finite segments according to well trajectory, then calculating from top to bottom with recursive algorithm. For the string shown as in Fig.1a), divide it into vertical section $\mathrm{AB}$, curved segment $\mathrm{BC}$ and deviated segment $\mathrm{CD}$, and establish calculation formula separately.

\subsection{Vertical section}

For the vertical string segment $\mathrm{AB}$, fluid pressure distributes symmetrically and the resultant force is zero on any axial micro-segment. So axial force is

$$
F_{x}=W-\gamma_{s} x A_{s}
$$

String weight is the only load. This formula can be used to the end of vertical section.

\subsection{Curved section}

If the curved section is close to circular arc, the calculation will be more convenient. As shown in Fig.1a), $S$ is variable of curvilinear coordinate.

Let $F_{s 0}$ string axial force at origin of curvilinear coordinate, $\alpha_{b}$ for the angle between string axis and gravity direction (abbreviated as inclination); $\alpha_{e}$ for the string inclination at the end of arc segment $(s=S)$. Then the curvature radius is $\rho=\frac{S}{\alpha_{e}-\alpha_{b}}$, the inclination at any arc length is $\alpha_{s}=\alpha_{b}+\frac{s}{\rho}$. Let $P_{s 0}$ for fluid pressure.

Intercept the string segment that the arc length is $s$, as shown in Fig.1b), then its forces from the effect of gravity and fluid pressure are buoyancy $s A_{o} \gamma_{o}$ (upwards) and gravity $s A_{s} \gamma_{s}$ (downwards).

The resultant forces in upper and lower cross sectional area by hydrostatic pressure is $P_{s 0} A_{o}$ and $\left[P_{s 0}+\gamma_{o} \rho\left(\sin \alpha_{s}-\sin \alpha_{b}\right)\right] A_{o}=F_{s} A_{o}$ respectively, along the pipe axis.

Actually, the forces in upper and lower area of the element are not fluid pressure, instead they are axial forces $F_{s 0}$ and $F_{s}$ respectively. By equilibrant principle, they have the following relationship

$$
\begin{aligned}
& {\left[F_{s 0}+P_{s 0} A_{o}+s A_{o} \gamma_{o}-s A_{s} \gamma_{s}\right] \cos \alpha_{s}-F_{s}-} \\
& {\left[P_{s 0}+\gamma_{o} p\left(\sin \alpha_{s}-\sin \alpha_{b}\right)\right] A_{o}=0}
\end{aligned}
$$

From which the axial force at $s$ is

$$
\begin{aligned}
& F_{s}=\left(F_{s 0}+P_{s 0} A_{o}+s A_{o} \gamma_{o}-s A_{s} \gamma_{s}\right) \cos \alpha_{s}- \\
& {\left[P_{s 0}+\gamma_{o} p\left(\sin \alpha_{s}-\sin \alpha_{b}\right)\right] A_{o}}
\end{aligned}
$$

Similarly, shearing force at $s$ is

$$
Q_{s}=\left(F_{s 0}+P_{s 0} A_{o}+s A_{o} \gamma_{o}-s A_{s} \gamma_{s}\right) \sin \alpha_{s}
$$

Thus it can be seen that fluid pressure may cause shearing force in curved string, the force points to direction as arc radial.

The two equations can be used until the end of the arc section BC, and so $F_{S}$ and $Q_{S}$ at point C can obtained.

If the curvature varies, the section should be divided into several parts, the above method should be used with each part.

\subsection{Deviated section}

The analysis method is similar to but simpler than the curved section, as shown in Fig.1a) and Fig.1c). Where $t$ is rectilinear coordinates, the inclination of deviated section is $\alpha_{e}$. Let $F_{t 0}\left(=F_{S}\right), Q_{t 0}\left(=Q_{S}\right), P_{t 0}$ for axial force, shearing force and fluid pressure at starting point $(t=0)$ respectively. Then, axial force and shearing force at any position $t$ are

$$
\begin{aligned}
& F_{t}=F_{t 0}-t A_{s} \gamma_{s} \cos \alpha_{e} \\
& Q_{t}=Q_{t 0}+t\left[A_{o} \gamma_{o}-A_{s} \gamma_{s}\right] \sin \alpha_{e}
\end{aligned}
$$

They both relate to the string inclination.

\subsection{Total buoyancy}

The resultant force of fluid pressure on vertical string section is zero. The resultant force of fluid pressure on curved string section is

$$
F_{f c}=S A_{o} \gamma_{o}+P_{s 0} A_{o} \cos \alpha_{b}-P_{S} A_{o} \cos \alpha_{e}
$$

Where $P_{S}=P_{s 0}+\gamma_{o} \rho\left(\sin \alpha_{e}-\sin \alpha_{b}\right)$. Let $\alpha_{b}=0$ substitute into above formula, then

$$
F_{f c}=S A_{o} \gamma_{o}+P_{s 0} A_{o}-\left(P_{s 0}+\gamma_{o} \rho \sin \alpha_{e}\right) A_{o} \cos \alpha_{e}
$$

Let $T$ for the length of deviated section, the buoyancy should be calculated in the following two instances in which the constraints of string lower end are different.

\subsubsection{The lower end is free}

There is fluid pressure on the lower end, but it doesn't exist in the cross section of origin. So buoyancy is

$$
F_{f T}^{1}=T A_{o} \gamma_{o}+P_{S} A_{o} \cos \alpha_{e}
$$


From formula (19) and formula (21), total buoyancy is $F_{f}=S A_{o} \gamma_{o}+P_{s 0} A_{o}+T A_{o} \gamma_{o}$. Considering $P_{s 0}=X A_{o} \gamma_{o}$ and $L=X+S+T$, then

$$
F_{f}=L A_{o} \gamma_{o}
$$

Formula (22) shows that although string deformation state is different in vertical wells from that in directional wells, the buoyancy the string gets is equal to the weight of fluid displaced by the string, upwards.

\subsubsection{The lower end is packed}

If there is no relative movement between string and packer, fluid pressure on string lower end is held by the packer, buoyancy exerted on string is

$$
F_{f t}^{2}=T A_{o} \gamma_{o}+P_{S} A_{o} \cos \alpha_{e}-P_{T} A_{o} \cos \alpha_{e}
$$

Consider that $P_{T}=P_{S}+\gamma_{o} T \cos \alpha_{e}$, then

$$
F_{f t}^{2}=T A_{o} \gamma_{o} \sin ^{2} \alpha_{e}
$$

Total buoyancy is

$$
\begin{aligned}
& F_{f}=s A_{o} \gamma_{o}+P_{s 0} A_{o}-\left(P_{s 0}+\gamma_{o} p \sin \alpha_{e}\right) A_{o} \cos \alpha_{e}+ \\
& T A_{o} \gamma_{o} \sin ^{2} \alpha_{e}
\end{aligned}
$$

When $\alpha_{e}=\pi / 2$, the results of formula (24) and formula (22) will be the same; or the results will be different.

There are two points should be noted in the previous analysis:

- The vertical string section does not directly get a resultant buoyancy force from fluid pressure; the fluid effect is passed to it in the form of axial force from lower string.

- Strings internal forces are different in deviated wells and in vertical wells. Firstly, resultant buoyancy force is different and so axial force distribution is different. Secondly, there is shearing force in string in deviated wells, but it doesn't exist in vertical wells.

Contact force of string with borehole wall is not considered in above calculation. Internal fluid pressure is also ignored to reduce complexity, when it is considered the analysis procedure is the same.

\section{Typical Engineering Cases}

\subsection{Example 1: Casing prestressing from pump- pressure}

In thermal recovery well, prestress the casing string is an effective method to promote casing safety. During cement curing, maintain a pump-pressure in casing string until cement sheath has formed. As results of pump- pressure, the piston effect elongates string and swelling effect shortens casing, but the total effect is that casing string is prolonged[8-10]. After wellhead is installed and pump-pressure is released, the elongation transforms into axial prestressing.

Given parameters as follows: casing outer diameter is $177.8 \mathrm{~mm}$, wall thickness is $9.19 \mathrm{~mm}$, casing length is $600 \mathrm{~m}$, Young's elastic modulus is $1.98 \times 10^{5} \mathrm{MPa}$, Poisson's ratio is 0.3 , pump-pressure is $16 \mathrm{MPa}$.

Calculation results: Casing axial prestressing by pump-pressure is $26.3 \mathrm{MPa}$.

\subsection{Example 2: Testing string deformation with permanent packer}

When testing of HPHT wells, permanent packers are often used. This packer permits lower end of string sliding freely in it but separates annular fluid pressure from formation pressure from below $[11,12]$. In different testing stages, string axial force and deformation are different.

Here, only consider the shut-in stage in which a inside tubing valve at string bottom is closed to separate internal pressure from formational pressure.

Given parameters as follows: outer and inner diameter of main tubing is $88.9 \mathrm{~mm}$ and $68.33 \mathrm{~mm}$ respectively, outer and inner diameter of slide pipe is $99.06 \mathrm{~mm}$ and $73.71 \mathrm{~mm}$ respectively, packer depth is $2800 \mathrm{~m}$, formation pressure is $60 \mathrm{MPa}$, fluid density in annulus is $1.5 \mathrm{~g} / \mathrm{cm}^{3}$, fluid density in string is $0.001 \mathrm{~g} / \mathrm{cm}^{3}$.

Calculation results: piston effect shorten the string with $0.69 \mathrm{~m}$, meanwhile, buckling effect shorten the string with $0.064 \mathrm{~m}$.

If the shut in valve is at wellhead, there will be a swelling effect that shortening the string with $0.66 \mathrm{~m}$.

\section{Summary}

The influence of fluid pressure on string depends mainly on two aspects, one is the pressure distribution determined by working environment and operation stage, the other is its spacial state and constraints. Calculation methods of string stress and deformation given by engineering handbooks doesn't distinguish these issues in detail. When calculating string stresses and deformation, method and formula should be selected carefully.

It is found that the traditional simplified buoyancy coefficient method, which is used to calculate string axial force and axial extension, can only be used in vertical wells with tubular string suspended freely, because in this condition buoyancy acts on the bottom of string.

If the string is constrained by downhole tools, such as packer or anchor, buoyancy could not be treated as usual. In directional well the buoyancy not only changes string axial force but induces shear stress in string cross section.

Examples from oil fields engineering indicate that when calculating the influence of fluid on string, operation sequence and constraints from borehole and downhole tools should be considered comprehensively. 


\section{References}

1. Gao Deli, Zhang Yuzhuo, Wang Jiaxiang, The Instability Theory and Control Technology in Underground Drilling and Digging Engineering. China Science Press, 217-225 (1999)

2. Liang Mingxi, High Temperature and High Pressure Well Testing Techniques. N. G. I 19, 1:76-79(1999)

3. Gao Bao Kui, Practical Model for Calculating Additional Loads on Casing String by High Temperature. O. D \& P. T 24, 1: 8-10(2002)

4. Drilling Manual (The First Party) Editorial Committee, Drilling Manual (The First Party) (Volume 1) , Beijing: Petroleum Industrial Press, 154-155(1999)

5. A.Lubinski, The Development of Drilling Engineering, Translated by Bao Youguang and Mei Jiang, Petroleum Industrial Press, Beijing(1999)

6. A.Lubinski, W.S.Althouse and J.L.Logan, Helical Buckling of Tubing Sealed in Packers. JPT, 655670(1962)

7. Mitchell R.F, Buckling Behavior of Well Tubing: The Packer Effect. S. P. E. J. SPE 9264,616624(1982)

8. Liu Wenzhang, Thermal Recovery Development Mode for Heavy Oil Reservoir, Beijing: Petroleum Industrial Press(1998)

9. Wang Zhaohui, Casing Damage Mechanism and Its Control in Thermal Recovery Wells in Northwestern Edge of Junggar Basin, Ph.D.Thesis, China University of Petroleum Beijing(2004)

10. G Maharaj, Thermal Well Casing Failure Analysis. SPE 36143, 651-655(1996)

11. E.Low, K.P.Seymourm, The Drilling and Testing of High-Pressure Gas Condensate Wells in the North Sea. SPE 17224(1998)

12. A.R.Davidson, G.Prise, C.French, Successful HighTemperature, High-Pressure Well Testing From a Semisubmersible Drilling Rig. SPE 23120(1991) 